\author{
A.I. Kupchishin $^{1}$, V.M. Lisitsyn ${ }^{2}$, M.N. Niyazov ${ }^{1}$, D.S. Utepova ${ }^{1 *}$ iD \\ ${ }^{1}$ Abai Kazakh National Pedagogical University, Almaty, Kazakhstan \\ ${ }^{2}$ Tomsk Polytechnic University, Tomsk, Russia \\ *e-mail: utepova_daniya@mail.ru

\section{Study of the mechanical properties of industrial plexiglas testing for flat straight bending}

\begin{abstract}
The mechanical properties of industrial plexiglas (polymethylmethacrylate) of various sizes (lengths, widths) have been studied when tested for plane straight bending with one concentrated force in the middle of the sample. By a result of the experimental work, the relationship between deformation and stress was obtained. It is clearly shown in the work that the experimental curves substantially depend on the parameters of the starting materials and are satisfactorily described by a linear model. The slope of tangent $(\alpha)$ for the curves ranges from 0,32 to $0,62 \% \mathrm{MPa}^{-1}$. The dependence of a $\alpha$ on the length and width of the samples changes significantly. Moreover, the maximum deformation varies in the range of $50-60 \%$, which confirms the dependence of the deformation properties of the material on horizontal components, in particular, on the length of the sample. The tensile strength of the material also differs between specimens of different sizes.
\end{abstract}

Key words: plexiglas, stress, deformation, mechanical properties, bending, elongation, strength.

\section{Introduction}

Polymer composite materials through to their unique mechanical properties are widely applied in various fields of industries and have a broad range of applications, particularly in every day life, construction, automotive and aerospace [1]. For example, the manufacture of parts using composite materials makes a significant contribution to reducing the weight of the final product, which undoubtedly affects its demand in the market. There is considerable of interest on the part of industrial industry and science lately in the research of composite materials for this reason. In tis important role played by the quality of the solids, of which products are manufactured. Before improving its parameters and becoming more attractive in today's competitive market, a significant number of companies conduct mechanical tests of materials to reveal their properties, as well as obtain new materials by mixing different components [2]. It is possible the find the interval of their use and prevent possible damage during use by examining the various characteristics of the products [3]. To study the mechanical properties of polymer composites are sundry kind of tests, including durability, tensile, compressive, tensile strength, elasticity, proportion- nality, impact strength and elongation after fracture and etc. [4]. Among these influences, the flexural test method has some advantages, such as easy sample preparation (fabrication) and no gripping, compared to tensile testing. The most common of these is straight bend. Straight bending is a traditional test method, which is conducted with the shear effects and local deformation when applied on supports of the load. The bending test is based on the application of a bending force to the test materials [5]. Test calculates the tensile strength at maximum bending load. There are different methods of bending test materials, such as methods of a concentrated force that was set in the middle of the sample between the supports and the two forces applied parallel on supports. The method of loading a test specimen with a solid force applied uniformly along the specimen between the supports and forces, and other methods. The single concentrated force method is the simplest and most affordable method for testing. In addition, to a certain extent, the results depend on the shape loading conditions and dimensions of the samples. In this work, we investigated the dependence of the deformation on the stress of industrial plexiglas when tested for flat straight bending by a focused one force [6]. 


\section{Research methods}

Industrial plexiglas was taken as the test material. The samples had thickness of $1 \mathrm{~mm}$, respectively, a width of $10 \mathrm{~mm}$ and $5 \mathrm{~mm}$ and were cut to different working lengths. The thickness of the polymethyl methacrylate samples was measured along the entire perimeter with a micrometer. The depending on the size made by a special cutting device which can be adjusted both the width and length of the sample material. The final touch in preparing a sample for testing is its grinding and polishing, which are designed to eliminate irregularities and burrs that appear as a result of cutting [7].

Figure 1 illustrates a block diagram of the experimental setup that was used to investigate the stress-strain relationship. The setup consists of the following parts: a force sensor (built-in voltage cap), strain sensor, the device for holding a sample (right and left supports,as well as cantilever clamps), an interface to output data. The components listed above are located on lower part of the BI - 50 machine, equipped with a mobile mechanism [8].

All experimental studies were carried out on attest setup providing a constant loading speed of 10 $\mathrm{mm} / \mathrm{min}$. the movement took place relative to the tip and supports, where the deflection was measured with an error of $\pm 5 \%$ and the load was $\pm 3 \%$. The convergence of the tip and supports held at a uniform speed. Plexiglas samples were loaded in the center of the sample with one concentrated force between the supports [9].

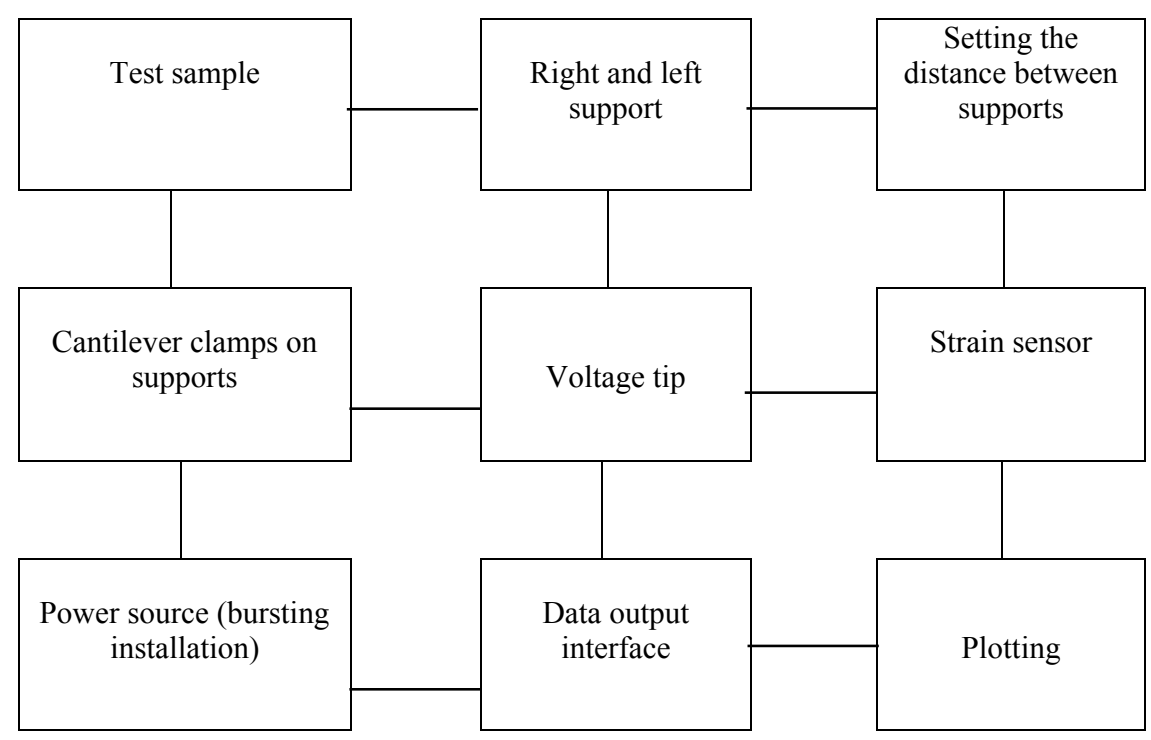

Figure 1 - Installation block diagram

The calculation of the dependence of $\varepsilon_{\mathrm{f}}$ on $\sigma_{\mathrm{f}}$ from the experimental data was performed by the following procedure. The flexural stress $\left(\sigma_{f}\right)$ at bending $\mathrm{z}$ is calculated be the formula below [10]. The stress $\left(\sigma_{\mathrm{f}}\right)$ takes into account the contribution of the horizontal component of the bending moment,

$$
\sigma_{\mathrm{f}}=\frac{3 \mathrm{FL}}{2 \mathrm{bh}^{2}}\left(1+\frac{4 \mathrm{z}^{2}}{\mathrm{~L}^{2}}\right) \text {, }
$$

where $\mathrm{b}$ - width of the specimen $(\mathrm{mm}), \mathrm{L}$ - working length, the distance between two support point $(\mathrm{mm}), \mathrm{h}$ - thickness of the specimen $(\mathrm{mm}), \mathrm{Z}$ represents the deflection of the material in the middle.

Elongation was calculated as the ratio of the deflection to the working length of the sample $(\varepsilon=$ $\mathrm{z} / \mathrm{L}$ ). Taking into account all values, we have [10]:

$$
\varepsilon_{f}=\frac{1}{2} \sqrt{\frac{2 \sigma_{\mathrm{f}} \mathrm{bh}^{2}}{3 \mathrm{FL}}-1}
$$

where $\mathrm{F}$ is bending force $(\mathrm{N}), \mathrm{b}$ and $\mathrm{h}$ are sample's dimensions. 


\section{Results and discussion}

As a result of experimental studies on testing samples of industrial plexiglass of various sizes for flat straight bending, the dependences of deformation or strain on stress were obtained, which are shown in Figures $2-5$. The figures mark the results of the experiment, and the lines mark the calculation data. The presentation of the physical and mechanical properties was carried out in the coordinates $\varepsilon, \sigma$. The relationship between stress $\sigma$ and strain $\varepsilon$ has no physical meaning, here stress $\sigma$ is an argument and strain $\varepsilon$ represents a function. It can be seen from the figures that the dependence of stress on deformation is satisfactorily described by a linear model for all sizes:

$$
\varepsilon=\alpha \sigma
$$

where $\varepsilon$ is deformation (\%), $\sigma$ is stress (MPa), $\alpha$ is the slope tangent of angle.

Figures $2-5$ show the slope of tangent for the curves, which ranges from 0.32 to $0.62 \% * \mathrm{MPa}^{-1}$.
The dependence of $\alpha$ on the length and width of the samples is shown in Table 1. It can be seen that with increasing length, the tangent of the angle increases for samples $10 \mathrm{~mm}$ wide. However, at a specimen length is $5 \mathrm{~mm}$, a stepwise increase occurs, i.e. strain $\alpha$ increases from 0.33 to $0.45 \% * \mathrm{MPa}^{-1}$, and then drops again to $0.32 \% * \mathrm{MPa}^{-1}$ and starts to increase again. From which it can be concluded that a decrease in the width of the samples affects the regularity and applicability of the linear model. A possible reason is that, due to its base in the form of acrylic resins, plexiglas is capable of producing microcracks, which at that time affect the uniform distribution of force over the entire area of the specimen. It is logical that cutting samples contributes to the appearance of microcracks, especially in cases of small sizes. Moreover, the maximum deformation varies in the range of $50-$ $60 \%$, which confirms the dependence of the deformation properties of the material on its horizontal components, in particular, on the length of the sample. The tensile strength of the material also differs between specimens of different sizes.

Table 1 - Dependence the targent of the angle of slope on the length and width of the sample

\begin{tabular}{|c|c|c|}
\hline Width $\mathrm{mm}$ & Length, mm & Targent of the angle, $\% * \mathrm{MPa}^{-1}$ \\
\hline 5 & 20 & 0,33 \\
\hline 5 & 30 & 0,41 \\
\hline 5 & 40 & 0,45 \\
\hline 5 & 50 & 0,35 \\
\hline 5 & 60 & 0,40 \\
\hline 5 & 70 & 0,46 \\
\hline 5 & 80 & 0,37 \\
\hline 10 & 20 & 0,41 \\
\hline 10 & 30 & 0,48 \\
\hline 10 & 40 & 0,50 \\
\hline 10 & 50 & 0,55 \\
\hline 10 & 60 & 0,57 \\
\hline 10 & 70 & 0,62 \\
\hline 10 & 80 & \\
\hline
\end{tabular}




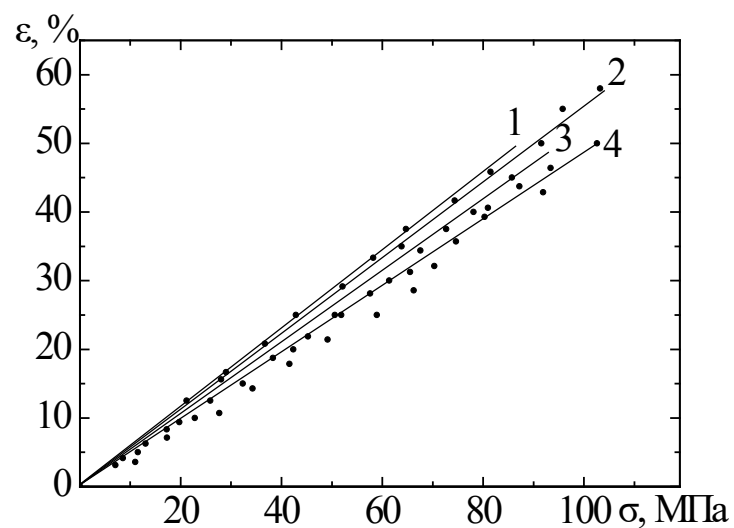

$1-80 ; 2-70 ; 3-60 ; 4-50 \mathrm{~mm}$

Calculation is represented by lines, experiment by figures;

Figure 2 - Represents the relationship between deformation and stress of an industrial plexiglas sample of various lengths and $10 \mathrm{~mm}$ width

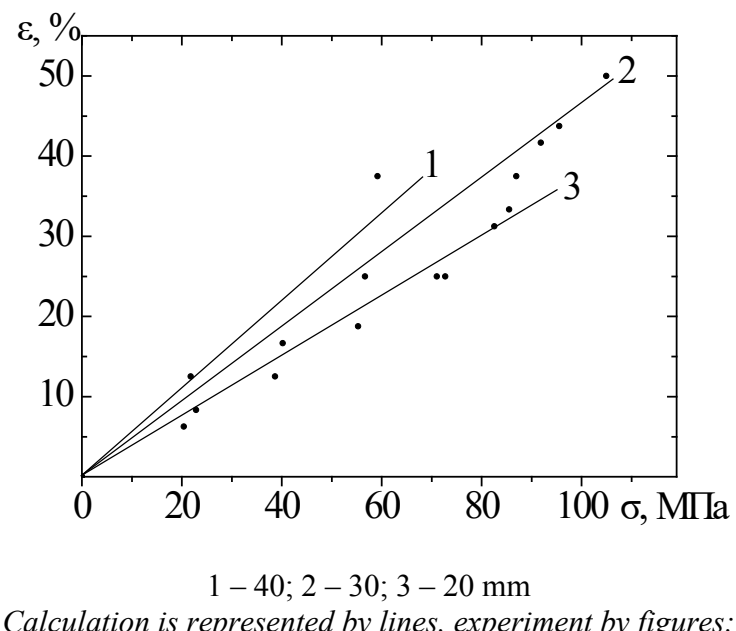

Calculation is represented by lines, experiment by figures;

Figure 3 - Represents the relationship between deformation and stress of an industrial plexiglas sample of various lengths and $10 \mathrm{~mm}$ width

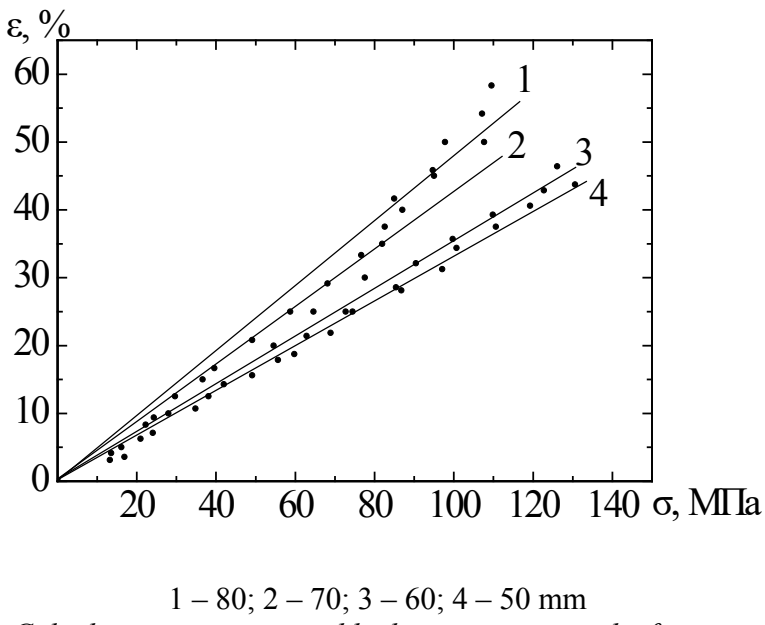

Calculation is represented by lines, experiment by figures;

Figure 4 - Represents the relationship between deformation and stress of an industrial plexiglas sample of various lengths and $5 \mathrm{~mm}$ width 


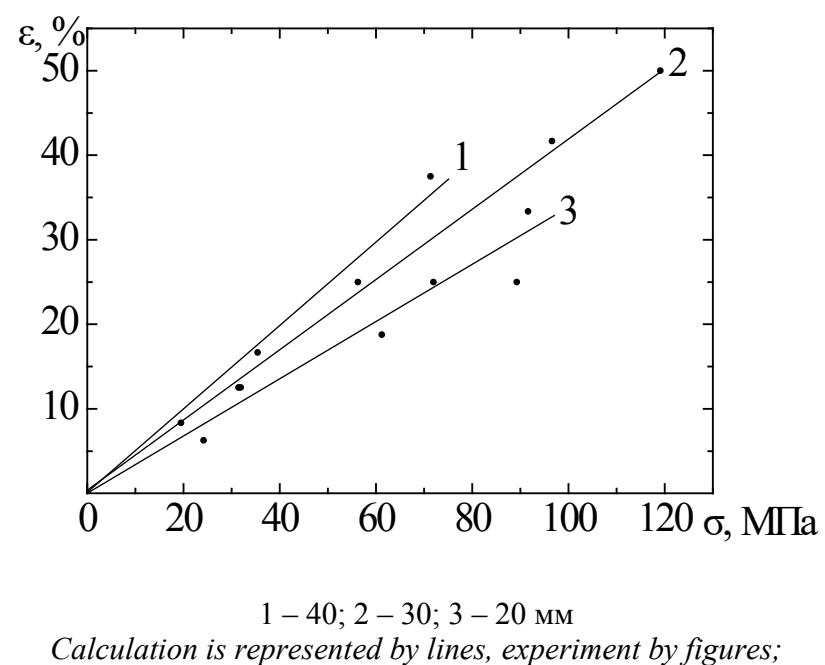

Figure 5 - Represents the relationship between deformation and stress of an industrial plexiglas sample of various lengths and $5 \mathrm{~mm}$ width

\section{Conclusions}

In the experimental work when tested on a flat straight bending ratio was found between strain and stress for industrial Plexiglas sample. It was found that the maximum deformation of materials of various sizes varies in the range of $50-60 \%$, which confirms the dependence of the deformation properties of the material on horizontal components, in particular the length of the sample.

The curves obtained after the calculation describing the relationship between elongation $\left(\varepsilon_{\mathrm{f}}\right)$ and flexural stress $\left(\sigma_{f}\right)$ are satisfactorily described by a linear model.

\section{References}

1 Gunay A., Fank S., Gulmez T., Durakbasa N.M. "Calculation of measurement uncertainty for plastic (ABS) material in flexural testing." International Journal of Metrology and Quality Engineering. Vol.4, Issue 1 (2013): 29.

2 Strobl G., Berlin: Springer-Verlag Berlin Heidelberg (2007)

3 Batu T., Lemu H. G. "Investigation of mechanical properties of false banan/glass fiber reinforced hybrid composite materials." Results in Materials, Vol. 8(2020).
4 Scutaru, M. L., Itu, C., Marin, M., Grif, H.Stefan. "Bending tests used to determine the mechanical properties of the components of a composite sandwich used in civil engineering." Procedia Manufacturing, Vol. 32 (2019): 259.

5 Al-Mosawi A. I., Ammash H.K. and Salaman A.J. Properties of Composite Materials Databook, Germany: Lambert Academic Publishing LAP, 2012.

6 Miller, Tara, ed. Introduction to Composites, 4th Edition. New York: Composites Institute, Society of the Plastics Industry,1998.

7 Kollar, L.P., Springer, G.S., Mechanics of Composite Structures. Cambridge: Cambridge U.P., 2003.

8 Cihan M. Sobey A., Blake J. "Mechanical and dynamic performance of woven flax/E-glass hybrid composites." Composites Science and Technology, Vol. 172 (2019): 36.

9 Abd El-baky M., Attia M., Abdelhaleem M., Hassan M. "Mechanical characterization of hybrid composites based on flax, basalt and glass fibers." Journal of Composite Materials, Vol. 54 (2020).

10 Voronova N.A, Kupchishin A.I, Niyazov M.N, Taipova BG, Abdukhairova A.T. "Reseach of electron irradiation effects upon plexiglas strain during bending test." IOP Conf. Series: Materials Science and Engineering, Vol.289 (2018):1. 